\title{
Contribución al conocimiento de las epífitas vasculares del Área Natural Protegida "Reserva Ecológica Sierra de Otontepec", Veracruz
}

Contribution to the knowledge of vascular epiphytes Protected Area "Reserva Ecologica Sierra Otontepec", Veracruz

Pérez-Lugo E. $\mathrm{R}^{1 凶}$, Alanís-Méndez J. L. ${ }^{1}$, Krömer T. ${ }^{2}$ y Chagoya-Fuentes J. L. ${ }^{3}$

${ }^{1}$ Universidad Veracruzana, Facultad de Ciencias Biológicas y Agropecuarias, Región Poza RicaTuxpan, Carretera Tuxpan-Tampico Kilómetro 7.5, Colonia Universitaria, 92850 Tuxpan, Veracruz, México. Tel: (783) 8344350 (783) 834 8979. ${ }^{2}$ Centro de Investigaciones Tropicales, Ex-Hacienda Lucas Martín, Privada de Araucarias s/n, Col. Periodistas, C.P. 91019. Xalapa, Veracruz, México.

${ }^{3}$ Instituto Nacional de Investigaciones Forestales, Agrícolas y Pecuarias. Campo Experimental Ixtlacuaco, Km. 4.5 Carretera Martínez De La Torre-Tlapacoyan, Col. Rojo Gómez Tlapacoyan, C.P. 93600. Martínez de la Torre, Veracruz.

${ }^{凶}$ Autor para correspondencia:perezlugo_15@hotmail.com

Recibido: $13 / 01 / 2014$

Aceptado: $14 / 07 / 2014$

\section{RESUMEN}

Se presenta una contribución de la diversidad de epifítas vasculares del ANP Sierra de Otontepec, en tres tipos de vegetación: bosque mesófilo de montaña, bosque tropical perennifolio y acahual, en los municipios de Chontla y Citlaltepetl. Se encontró que este grupo de plantas está representado principalmente por las familias Bromeliaceae (26.4\%) y Polypodiaceae (23.5\%), seguido de la familia Orchidaceae y Piperacea (14.7\%). El BMM presento mayor riqueza de especies (46\%), mientras que el BTP presento menor riqueza (23\%) en comparación con el AC (31\%). Se registraron dos especies en la NOM-059-SEMARNAT-2010, en la categoría de Amenazadas (Tillandsia imperialis y Prostecheae mariae). Este grupo de plantas se ven afectadas por la fragmentación y recolección ilegal, por lo que, se considera necesario generar conocimiento sobre las especies que se encuentran en la reserva.

Palabras clave: Epifitas vasculares, Conservación, Reserva Ecológica, Sierra de Otontepec.

\section{ABSTRACT}

We present a contribution to the diversity of epiphytic vascular Protected Area Sierra de Otontepec in three vegetation types: cloud forest, tropical rain forest and acahual, in the municipalities of Chontla and Citlaltepetl. We found that this group of plants is mainly represented by the Bromeliaceae family

\section{Revista Científica Biológico Agropecuaria Tuxpan 2 (1)}


(26.4\%) and Polypodiaceae (23.5\%), followed by the family Orchidaceae and Piperacea (14.7\%). The BMM showed higher species richness (46\%), while the present BTP less wealth (23\%) compared with AC (31\%). There were two species in the NOM-059-SEMARNAT-2010, in the category of threatened (Tillandsia imperialis and Prostecheae marine). This group of plants is affected by fragmentation and illegal collection, so it is considered necessary to generate knowledge about the species found in the reserve.

Keywords: Vascular epiphytes, Conservation, Ecological Reserve, Sierra de Otontepec.

\section{INTRODUCCIÓN}

Las epífitas vasculares han evolucionado en 73 familias y 912 géneros que incluyen al menos una especie epífita (Zotz, 2013), muchas de ellas representan especies aisladas o géneros en familias de otros hábitos terrestres. Sin embargo, en sólo cuatro familias; Orchidaceae, Araceae, Bromeliaceae y Piperaceae, así como en las Pteridofitas, se encuentran más del $80 \%$ de las especies de epífitas (Kress, 1986; Gentry y Dodson, 1987), calculando 27, 500 especies con hábito epífito que representan aproximadamente el 9\% de las plantas vasculares (Zotz, 2013). En México, las epífitas son uno de los componentes más atractivos e interesantes de nuestras selvas y bosques. De forma preliminar se registraron para el país cerca de 1,207 especies, distribuidas en 37 familias y 174 géneros (Aguirre-León, 1992). Posteriormente Wolf y Flamenco (2003) registraron 1,173 especies solo para el estado de Chiapas, incrementando esta cifra a 1,377 especies epifitas para el país. En el centro de Veracruz existen al menos 604 especies de plantas epífitas (Flores-Palacios et al., 2011) y para el norte de Veracruz se tienen registros de epífitas de las familias Bromeliaceae, Cactaceae y Orchidaceae en 3 familias, 5 géneros y 10 especies (AlanísMéndez et al., 2007). Las epifitas son consideradas elementos diversos e importantes de los bosques tropicales, pero como organismos del dosel, son muy vulnerables a la deforestación y fragmentación (Barthlott et al., 2001; Wolf, 2005; Krömer et al., 2007; FloresPalacios y García-Franco, 2008; Köster et al.,
2009). La tendencia actual de extracción desmedida y destrucción de su hábitat, ha provocado la reducción de sus poblaciones, poniéndose en riesgo de extinción a muchas de éstas (Flores-Palacios y Valencia-Díaz, 2007; Haeckel, 2008). Por lo tanto, el objetivo de este estudio es generar conocimiento acerca de su composición florística y estado de conservación en la Reserva Ecológica "Sierra de Otontepec", Veracruz. Así mismo, se propone generar información sobre especies que actualmente se encuentran bajo estatus de protección con base a la legislación ambiental mexicana (NOM059-SEMARNAT-2010).

\section{MATERIALES Y MÉTODOS}

La Sierra de Otontepec se localiza en la zona norte de la entidad veracruzana, entre las coordenadas $97^{\circ} 58^{\prime} 30^{\prime \prime}$ y $97^{\circ} 48^{\prime} 00^{\prime \prime}$ de longitud Oeste, y $21^{\circ} 19^{\prime} 19^{\prime \prime}$ y $21^{\circ} 09^{\prime} 34^{\prime \prime}$ de latitud Norte aproximadamente, comprende una superficie de 15,152-00-00.00 hectáreas, donde incluye los Municipios de Ixcatepec, Tepetzintla, Chontla, Citlaltépetl, Tantima, Tancoco, Cerro Azul y Chicontepec (SEDESMA, 2007).

Se seleccionaron dos sitios para muestreo en los municipios de Chontla y Citlaltepetl que conforman la Reserva Ecológica Sierra de Otontepec, donde se muestrearon tres tipos de vegetación; bosque mesófilo de montaña (BMM), bosque tropical perennifolio (BTP) y acahual (AC). El muestreo para el inventario de epífitas vasculares se basa en el método propuesto por 
Gradstein et al. (2003), estableciendo cuatro parcelas no permanentes de 20 × $20 \mathrm{~m}$ (400 $\mathrm{m}^{2}$ ), ubicadas niveles altitudinales mayores a $300 \mathrm{msnm}$ que es el límite de la reserva y dependiendo de las condiciones del acceso al bosque, por cada tipo de vegetación. Así mismo, se efectuaron transectos en diferentes puntos del bosque conservado y secundario como complemento al inventario.

\section{RESULTADOS}

Se registraron 34 especies en 19 géneros y 7 familias con epífitas vasculares en vegetación de BMM, BTP y AC de la Reserva Ecológica Sierra de Otontepec, presentando mayor riqueza de especies las familias Bromeliaceae (9 spp.; 26.4\%) y Polypodiaceae (8 spp.; $23.5 \%$ ) seguido de Orchidaceae y Piperacea (5 spp.; 14.7\%) y Araceae (4 spp.; 11.7\%). De los ambientes conservados, el BMM presento mayor riqueza de especies (22 spp.; 46\%), mientras que el BTP presento menor riqueza (11 spp.; 23\%) en comparación con el ambiente secundario AC (15 spp.; 31\%). Se registraron dos especies en categoría de Amenazadas (A) en la NOM-059SEMARNAT-2010; Prosthechea mariae y Tillandsia imperialis de las cuales $P$. mariae es endémica para México.

\section{DISCUSIÓN}

Se cuenta actualmente con el registro de 34 especies de epífitas vasculares que representan el $5.6 \%$ del total que se tiene registrado para el centro de Veracruz (FloresPalacios et al., 2011). En este estudio se encontraron 8 especies como nuevo registro para la Sierra de Otontepec, con base a lo que se tienen para la región en Castillo-Campos y Medina-Abreo (1996); SEDESMA (2007) y Alanís-Méndez et al. (2007). Las familias mejor representadas en riqueza de especies son las bromelias y helechos con nueve y ocho especies respectivamente, seguido de las orquídeas y piperáceas con 5 especies, taxones que son características de la flora epífita de los bosques tropicales (Kress, 1986; Gentry y Dodson, 1987), ya que tan solo en este estudio representan el $79 \%$ de la riqueza epifítica vascular de la reserva. El BTP presento menor riqueza, dado que solo se registraron 11 especies en comparación con el BMM donde se encontraron 22 especies, sin embargo, en el acahual se encontraron 15 especies en áreas que corresponde a fragmentos continuos del BTP, por lo tanto, tal riqueza podría estar relacionada con la conectividad de la vegetación, lo que podría favorecer especies de permanencia (Castillo-Campos et al., 2008). Hietz y HietzSeifert (1995), mencionan que la diversidad de hábitats puede contribuir a la diversidad de epífitas en bosques húmedos, pero la importancia de este efecto no se puede distinguir de la influencia del clima, lo cual explica la riqueza en número de especies epifitas en el bosque mesófilo.

Las dos especies de las familias Bromeliaceae (Tillandsia imperialis) y Orchidaceae (Prosthechea mariae) que se encontraron en la NOM-059-SEMARNAT2010 en la categoría de Amenazadas (SEMARNAT, 2013), son de importancia económica ya que son comercializadas como plantas de ornato para jardín o en rituales religiosos (Mondragón, 2008; Mondragón y Villa-Guzmán, 2008). Flores-Palacios y Valencia-Díaz (2007), estiman que alrededor del $25 \%$ de la riqueza de epífitas y el $47 \%$ de las orquídeas epífitas en el Estado de Veracruz son objeto de comercio ilegal. Hietz y HietzSeifert, (1994) y Espejo-Serna y colaboradores (2010), describen a Tillandsia imperialis como especie endémica de México de bosque mesófilo de montaña y bosque de encino-pino entre los 1500 y $3000 \mathrm{msnm}$, con poblaciones abundantes que al parecer no presentan problemas de supervivencia sin embargo en el área de estudio se encontró en bosque mesófilo alrededor de los $1100 \mathrm{msnm}$, con poca presencia de individuos. La orquídea

Prosthechea mariae García-Cruz y 
colaboradores en el 2003, la describen en bosque mesófilo de montaña del noreste de Querétaro, así como en cañadas húmedas en medio del bosque de encino y la consideran como un elemento raro y en consecuencia vulnerable a la extinción, en la Reserva Ecológica de Otontepec, se observó en acahuales y en vegetación ríparia entre los 400 a $500 \mathrm{msnm}$.

\section{CONCLUSIONES}

La riqueza de epífitas en la Reserva Ecológica de la Sierra de Otontepec, está representada por las bromelias, helechos, orquídeas y piperaceas principalmente, ya que son las que muestran mayor riqueza de especies.

De acuerdo a los muestreos en los tres tipos de vegetación (BMM, BTP y AC), el $B M M$ es el que presenta mayor diversidad en especies.

Dos de las especies que se enlistaron en el inventario se encuentran en la NOM-059SEMARNAT-2010 en la categoría de amenazadas.

El conocimiento de la biodiversidad, son fundamentales para el desarrollo de un país, por lo que la biodiversidad debe ser conservada y aprovechada adecuadamente, principalmente en áreas naturales protegidas consideradas como reservas de especies ecológicamente importantes.

\section{LITERATURA CITADA}

Aguirre-León, E. 1992. Vascular epiphytes of Mexico: a preliminary inventory. Selbyana, 13: 72-76.

Alanís-Méndez, J. L., Muñoz, A. F. O, López, O. M., Cuervo, L. L. y Raya, C. B. E. 2007. Aportes al conocimiento de las epífitas (Bromeliaceae, Cactaceae y Orchidaceae) en dos tipos de vegetación del Municipio de Pánuco, Veracruz, México. UDO Agrícola, 7 (1): 160-174.

Barthlott, W., Schmit-Neuerburg, V., Nieder, J. y Engwald S. 2001. Diversity and abundance of vascular epiphytes: a comparison of secondary vegetation and primary montane rain forest in the Venezuelan Andes. Plant Ecology, 152: 145-156.

https://doi.org/10.1023/a:1011483901452

Castillo-Campos, G. y Medina, A. M. 1996. La vegetación de la Sierra de Tantima Otontepec, Veracruz, México, La Ciencia y El Hombre, 24: 45-67.

Castillo-Campos G., Halfter, G. y Moreno, C. E. 2008. Primary and secondary vegetation patches as contributors to floristic diversity in a tropical deciduous forest landscape. Biodivers Conserv, 17: 1701-1714. https://doi.org/10.1007/s10531-008-9375-7

Flores-Palacios, A. y García-Franco, J. G. 2008. Habitat isolation changes the beta diversity of the vascular epiphyte community in lower montane forest, Veracruz, Mexico. Biodiversity and Conservation, 17: 191-207. https://doi.org/10.1007/s10531-007-9239-6

Flores-Palacios, A. y Valencia-Díaz, S. 2007. Local illegal trade reveals unknown diversity and involves a high species richness of wild vascular epiphytes. Biological Conservation, 136: 372-387. https://doi.org/10.1016/j.biocon.2006.12.017

Flores-Palacios, A., García-Franco, J. G., Valencia-Díaz, S., Solís-Montero, L. y Cruz-Angón, A. 2011. Diversidad y

conservación de plantas epífitas vasculares en el Centro del Estado. En La biodiversidad en Veracruz: Estudio de Estado, vol. I, Comisión Nacional para el Conocimiento y Uso de la Biodiversidad, México. pp. 493-501. https://doi.org/10.5962/bhl.title.118977 
García-Cruz, J., Sánchez-Saldaña, L. M., Jiménez-Machorro, R. y Solano-Gómez, R. 2003. Orchidaceae. Tribu Epidendreae. Flora del Bajío y de Regiones Adyacentes. Fascículo 119. Instituto de Ecología, A.C. Centro Regional del Bajío Patzcuaro, Michoacán México. 178 p. https://doi.org/10.22201/ib.9786073009102e.2018

Gentry, A. H. y Dodson, C. H. 1987. Diversity and biogeography of neotropical vascular epiphytes. Annals of the Missouri Botanical, 74: 205-233. https://doi.org/10.2307/2399395

Gradstein, S. R., Nadkarni, N. M., Krömer T., Holz, I. y Nöske, N. 2003. A protocol for rapid and representative sampling of vascular and non-vascular epiphyte diversity in tropical rain forests. Selbyana, 24: 105-111.

Haeckel, I. B. 2008. The "arco floral": ethnobotany of Tillandsia and Dasylirion spp. in a Mexican religious adornment. Economic Botany, 62: 90-95. https://doi.org/10.1007/s12231-008-9009-8

Hietz, P. y Hietz-Seifert, U. 1995. Composition and ecology of vascular epiphyte communities along an altitudinal gradient in central Veracruz, Mexico. J. Veg. Sci., 6: 487-498.

https://doi.org/10.14198/cdbio.1999.01.02

Hietz, P. y Hietz-Seifert, U. 1994. Epífitas de Veracruz, Guía Ilustrada para las Regiones de Xalapa y los Tuxtlas, Veracruz. Instituto de Ecología, AC. México. 236 p. https://doi.org/10.2307/3236347

Köster, N., Friedrich, K., Nieder, N. y Barthlott, W. 2009. Conservation of epiphyte diversity in an Andean landscape transformed by human land use. Conservation Biology, 25: 911-919. https://doi.org/10.1111/j.1523-1739.2008.01164.x

Kress, W. J. 1986. The systematic distribution of vascular epiphytes: an update. Selbyana, 9: 2-22.
Krömer, T., Gradstein, S. R. y Acebey, A. 2007. Diversidad y ecología de epífitas vasculares en bosques montanos primarios y secundarios de Bolivia. Ecología en Bolivia, 42(1): 23-33. https://doi.org/10.21311/51.1.5

Mondragón, D. y Villa-Guzmán, D. M. 2008. Estudio etnobotánico de las bromelias epifitas en la comunidad de Santa Catarina Ixtepeji, Oaxaca, México. Polibotánica, 26: 175-191.

SEDESMA (Secretaria de Desarrollo Social y Medio Ambiente). 2007. Programa de Manejo de la Reserva Ecológica Sierra de Otontepec. Secretaria de Desarrollo Social y Medio Ambiente. Gobierno del Estado de Veracruz. 213 p. https://doi.org/10.2307/j.ctvckq2rp.15

SEMARNAT (Secretaria de Medio Ambiente y Recursos Naturales). 2013. NOM-059SEMARNAT-2010. Protección ambiental -Especies nativas de México de flora y fauna silvestre-Categorías de riesgo $\mathrm{y}$ especificaciones para su inclusión, exclusión cambio- Lista de especies en riesgo. Diario Oficial de la Federación. https://doi.org/10.3989/graellsia.2012.v68.063

Wolf, J. H. D. 2005. The response of epiphytes to anthropogenic disturbance of pine oak forests in the highlands of Chiapas, Mexico. Forest Ecology and Management, 212: 376-393. https://doi.org/10.1016/j.foreco.2005.03.027

Wolf, J. H. y Flamenco, A. 2003. Patterns in species richness and distribution of vascular epiphytes in Chiapas, Mexico. Journal of Biogeography, 30: 1689-1707. https://doi.org/10.1046/j.1365-2699.2003.00902.x

Zotz, G. 2013. The systematic distribution of vascular epiphytes - a critical update. Botanical Journal of the Linnean Society, 171: 453-481. https://doi.org/10.1111/boj.12010 
Pérez et al., 2014

Copyright (c) 2014 G. E. R. Pérez Lugo, J. L. Alanis Méndez, T. Krömer y J. L. Chag oya Fuentes

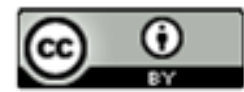

Este texto está protegido por una licencia Creative Commons 4.0.

Usted es libre para Compartir —copiar y redistribuir el material en cualquier medio o formato- y Ad aptar el documento —remezclar, transformar y crear a partir del material一 para cualquier propósito, inchso para fines comerciales, siempre que cumpla la condición de:

Atribución: Usted debe dar crédito a la obra original de manera adecuada, proporcionar un enlace a la licencia, e indicar si se han realizado cambios. Puede hacerlo en cualquier forma tazonable, pero no de forma tal que sugiera que tiene el apoyo del licenciante o lo recibe por el usoque hace de la obra.

Resumendelicencia - Textocompletodklalicerria 